\title{
NHS
}

Leadership Academy

\section{Leadership Framework A Summary}


(C) 2011 NHS Leadership Academy. All rights reserved.

The Leadership Framework is published on behalf of the NHS Leadership Academy by NHS Institute for Innovation and Improvement, Coventry House, University of Warwick Campus, Coventry, CV4 7AL.

Publisher: NHS Institute for Innovation and Improvement, Coventry House, University of Warwick Campus, Coventry, CV4 7AL.

This publication may be reproduced and circulated free of charge for non-commercial purposes only by and between NHS-funded organisations in England, Scotland, Wales and Northern Ireland staff, and their related networks and officially contracted third parties. This includes the right to reproduce, distribute and transmit this publication in any form and by any means, including e-mail, photocopying, microfilming, and recording. No other use may be made of this publication or any part of it except with the prior written permission and application for which should be in writing and addressed to the Director of Leadership (and marked 're. permissions'). Written permission must always be obtained before any part of this publication is stored in a retrieval system of any nature, or electronically. Reproduction and transmission of this publication must be accurate, must not be used in any misleading context and must always be accompanied by this Copyright Notice.

Warning: Unauthorised copying, storage, reproduction, adaptation or other use of this publication or any part of it is strictly prohibited. Doing an unauthorised act in relation to a copyright work may give rise to civil liabilities and criminal prosecution.

The Clinical Leadership Competency Framework was created with the agreement of the NHS Institute for Innovation and Improvement and the Academy of Medical Royal Colleges from the Medical Leadership Competency Framework which was created, developed and is owned jointly by the NHS Institute for Innovation and Improvement and the Academy of Medical Royal Colleges.

NHS Institute for Innovation and Improvement and Academy of Medical Royal Colleges (2010) Medical Leadership Competency Framework, 3rd edition, Coventry: NHS Institute for Innovation and Improvement.

(c) NHS Institute for Innovation and Improvement and Academy of Medical Royal Colleges 2010 


\section{The Leadership Framework}

The Leadership Framework provides a consistent approach to leadership development for staff in health and care irrespective of discipline, role or function, and represents the foundation of leadership behaviour that all staff should aspire to. Fundamental to its development was a desire to build on existing leadership frameworks used by different staff groups and create a single overarching leadership framework for all staff in health and care.

In developing the Leadership Framework detailed research and consultation with a wide cross section of staff and stakeholders has been undertaken, including those with a patient perspective and those involved in healthcare outside the NHS such as professional bodies, academics, regulators and policy makers.

Those consulted embraced the concept of the Leadership Framework because it affords a common and consistent approach to professional and leadership development, based on shared values and beliefs which are consistent with the principles and values of the NHS Constitution?

The Leadership Framework is based on the concept that leadership is not restricted to people who hold designated leadership roles and where there is a shared responsibility for the success of the organisation, services or care being delivered. Acts of leadership can come from anyone in the organisation and as a model it emphasises the responsibility of all staff in demonstrating appropriate behaviours, in seeking to contribute to the leadership process and to develop and empower the leadership capacity of colleagues².

This document provides a summary of the seven domains of the Leadership Framework. A full and web based version can be found at www.leadershipacademy.nhs.uk/lf

\section{Design and structure of the Leadership Framework}

Delivering services to patients, service users, carers and the public is at the heart of the Leadership Framework. The needs of the people who use services have always been central to healthcare and all staff work hard to improve services for them. However, if we are going to transform services, acting on what really matters to patients and the public is essential and involves the active participation of patients, carers, community representatives, community groups and the public in how services are planned, delivered and evaluated ${ }^{3}$.

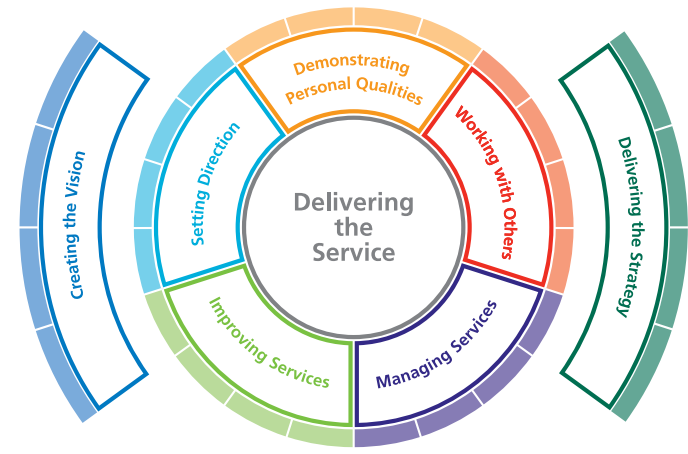

The Leadership Framework is comprised of seven domains. Within each domain there are four categories called elements and each of these elements is further divided into four descriptors. These statements describe the leadership behaviours, which are underpinned by the relevant knowledge, skills and attributes all staff should be able to demonstrate.

To improve the quality and safety of health and care services, it is essential that staff are competent in each of the five core leadership domains shown at right: demonstrating personal qualities, working with others, managing services, improving services, and setting direction. The two other domains of the Leadership Framework, creating the vision and delivering the strategy, focus more on the role and contribution of individual leaders and particularly those in senior positional roles.

\footnotetext{
${ }^{1}$ Department of Health (2010) The NHS Constitution: the NHS belongs to us all. The NHS Constitution can be accessed via http://www.nhs.uk/choiceintheNHS/Rightsandpledges/NHSConstitution/Pages/Overview.aspx

${ }^{2}$ NHS Institute for Innovation and Improvement and Academy of Medical Royal Colleges (2009) Shared Leadership: Underpinning of the MLCF

${ }^{3}$ Patient and Public Engagement, Department of Health (2009) Putting Patients at the Heart of Care: The Vision for Patient and Public Engagement in Health and Social Care. www.dh.gov.uk/ppe
} 
The word 'patient' is used throughout the Leadership Framework to generically cover patients, service users, and all those who receive healthcare. Similarly, the word 'other' is used to describe all colleagues from any discipline and organisation, as well as patients, service users, carers and the public.

\section{The leadership context}

The application and opportunity to demonstrate leadership will differ and the context in which competence can be achieved will become more complex and demanding with career progression. We have therefore used four stages to describe this and to help staff understand their progression and development as a leader. They are:

Stage 1 Own practice/immediate team - is about building personal relationships with patients and colleagues, often working as part of a multi-disciplinary team. Staff need to recognise problems and work with others to solve them. The impact of the decisions staff take at this level will be limited in terms of risk.

Stage 2 Whole service/across teams - is about building relationships within and across teams, recognising problems and solving them. At this level, staff will need to be more conscious of the risks that their decisions may pose for self and others for a successful outcome.

Stage 3 Across services/wider organisation - is about working across teams and departments within the wider organisation. Staff will challenge the appropriateness of solutions to complex problems. The potential risk associated with their decisions will have a wider impact on the service.

Stage 4 Whole organisation/healthcare system - is about building broader partnerships across and outside traditional organisational boundaries that are sustainable and replicable. At this level leaders will be dealing with multi-faceted problems and coming up with innovative solutions to those problems. They may lead at a national/international level and would be required to participate in whole systems thinking, finding new ways of working and leading transformational change. Their decisions may have significant impact on the reputation of the NHS and outcomes and would be critical to the future of the NHS. 


\section{Application of the Leadership Framework and supporting tools}

The Leadership Framework is designed to enable staff to understand their progression as a leader and to support fostering and developing talent. There are many ways it can be applied, for example:

- To raise awareness that effective leadership is needed across the whole organisation

- To underpin a talent management strategy

- As part of an existing leadership development programme

- To inform the design and commissioning of training and development programmes

- To develop individual leadership skills

- As part of team development

- To enhance existing appraisal systems

- To inform an organisation's recruitment and retention processes.

To assist users the full and web based version have a suite of indicators across a variety of work place situations which illustrate the type of activity staff could be demonstrating relevant to each element and stage as well as examples of behaviours if they are not.

\section{Supporting tools}

A self assessment and $360^{\circ}$ feedback tool support the Leadership Framework; in addition an online development module signposts development opportunities for each of the seven domains. The $360^{\circ}$ is a powerful tool to help individuals identify where their leadership strengths and development needs lie. The process includes getting confidential feedback from line managers, peers and direct reports. As a result, it gives an individual an insight into other people's perceptions of their leadership abilities and behaviour.

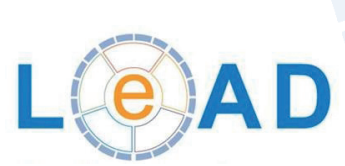

To assist with integrating the competences into postgraduate curricula and learning experiences, there is the LeAD e-learning resource which is available on the National Learning Management System and through e-Learning for Healthcare (www.e-lfh.org.uk/LeAD).

The Clinical Leadership Competency Framework and the Medical Leadership Competency Framework are also available to specifically provide staff with clinically based examples in practice and learning and development scenarios across the five core domains shared with the Leadership Framework.

A summary version of the Leadership Framework follows, which includes the domains, elements and descriptors. Work-place indicators that demonstrate the practical application of the framework at the four stages are included as tables in the back of the document. The examples in practice are not included, however these are available in the full document as well as on the website (www.leadershipacademy.nhs.uk/lf). 


\section{Demonstrating Personal Qualities}



Effective leadership requires individuals to draw upon their values, strengths and abilities to deliver high standards of service. To do so, they must demonstrate effectiveness in:

- Developing self awareness by being aware of their own values, principles, and assumptions, and by being able to learn from experiences

- Managing yourself by organising and managing themselves while taking account of the needs and priorities of others

- Continuing personal development by learning through participating in continuing professional development and from experience and feedback

- Acting with integrity by behaving in an open, honest and ethical manner.

\subsection{Developing self awareness}

1. Recognise and articulate their own value and principles, understanding how these may differ from those of other individuals and groups

2. Identify their own strengths and limitations, the impact of their behaviour on others, and the effect of stress on their own behaviour

3. Identify their own emotions and prejudices and understand how these can affect their judgement and behaviour

4. Obtain, analyse and act on feedback from a variety of sources

\subsection{Managing yourself}

1. Manage the impact of their emotions on their behaviour with consideration of the impact on others

2. Are reliable in meeting their responsibilities and commitments to consistently high standards

3. Ensure that their plans and actions are flexible, and take account of the needs and work patterns of others

4. Plan their workload and activities to fulfil work requirements and commitments, without compromising their own health

\subsection{Continuing personal development}

1. Actively seek opportunities and challenge for personal learning and development

2. Acknowledge mistakes and treat them as learning opportunities

3. Participate in continuing professional development activities

4. Change their behaviour in the light of feedback and reflection

\subsection{Acting with integrity}

1. Uphold personal and professional ethics and values, taking into account the values of the organisation and respecting the culture, beliefs and abilities of individuals

2. Communicate effectively with individuals, appreciating their social, cultural, religious and ethnic backgrounds and their age, gender and abilities

3. Value, respect and promote equality and diversity

4. Take appropriate action if ethics and values are compromised 


\section{Working with Others}

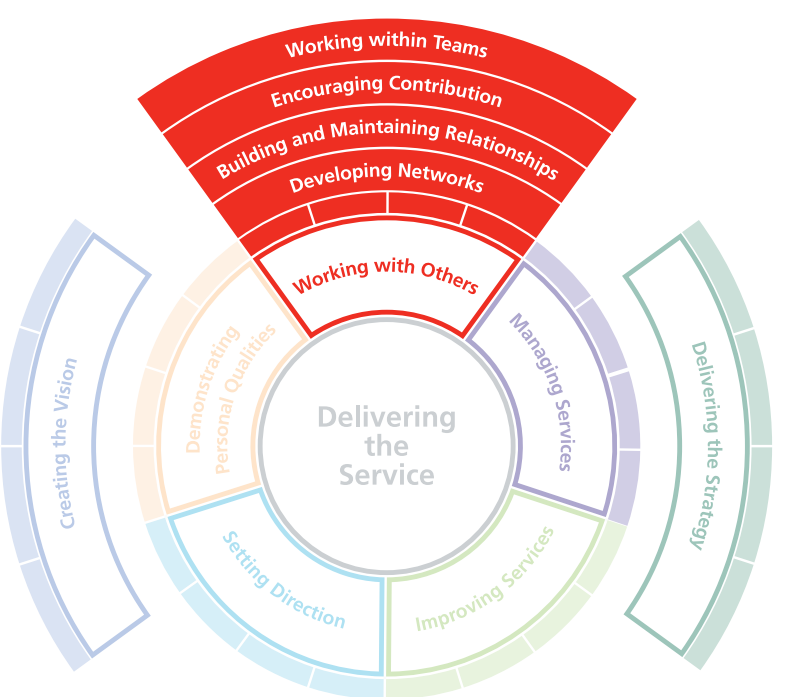

Effective leadership requires individuals to work with others in teams and networks to deliver and improve services. To do so, they must demonstrate effectiveness in:

- Developing networks by working in partnership with patients, carers, service users and their representatives, and colleagues within and across systems to deliver and improve services

- Building and maintaining relationships by listening, supporting others, gaining trust and showing understanding

- Encouraging contribution by creating an environment where others have the opportunity to contribute

- Working within teams to deliver and improve services.

\subsection{Developing networks}

1. Identify opportunities where working with patients and colleagues in the clinical setting can bring added benefits

2. Create opportunities to bring individuals and groups together to achieve goals

3. Promote the sharing of information and resources

4. Actively seek the views of others

2.2 Building and maintaining relationships

1. Listen to others and recognise different perspectives

2. Empathise and take into account the needs and feelings of others

3. Communicate effectively with individuals and groups, and act as a positive role model

4. Gain and maintain the trust and support of colleagues

\subsection{Encouraging contribution}

1. Provide encouragement, and the opportunity for people to engage in decision-making and to challenge constructively

2. Respect, value and acknowledge the roles, contributions and expertise of others

3. Employ strategies to manage conflict of interests and differences of opinion

4. Keep the focus of contribution on delivering and improving services to patients

\subsection{Working within teams}

1. Have a clear sense of their role, responsibilities and purpose within the team

2. Adopt a team approach, acknowledging and appreciating efforts, contributions and compromises

3. Recognise the common purpose of the team and respect team decisions

4. Are willing to lead a team, involving the right people at the right time 


\section{Managing Services}



Effective leadership requires individuals to focus on the success of the organisation(s) in which they work. To do so, they must be effective in:

- Planning by actively contributing to plans to achieve service goals

- Managing resources by knowing what resources are available and using their influence to ensure that resources are used efficiently and safely, and reflect the diversity of needs

- Managing people by providing direction, reviewing performance, motivating others, and promoting equality and diversity

- Managing performance by holding themselves and others accountable for service outcomes.

\subsection{Planning}

1. Support plans for clinical services that are part of the strategy for the wider healthcare system

2. Gather feedback from patients, service users and colleagues to help develop plans

3. Contribute their expertise to planning processes

4. Appraise options in terms of benefits and risks

\subsection{Managing resources}

1. Accurately identify the appropriate type and level of resources required to deliver safe and effective services

2. Ensure services are delivered within allocated resources

3. Minimise waste

4. Take action when resources are not being used efficiently and effectively

\subsection{Managing people}

1. Provide guidance and direction for others using the skills of team members effectively

2. Review the performance of the team members to ensure that planned services outcomes are met

3. Support team members to develop their roles and responsibilities

4. Support others to provide good patient care and better services

\subsection{Managing performance}

1. Analyse information from a range of sources about performance

2. Take action to improve performance

3. Take responsibility for tackling difficult issues

4. Build learning from experience into future plans 


\section{Improving Services}

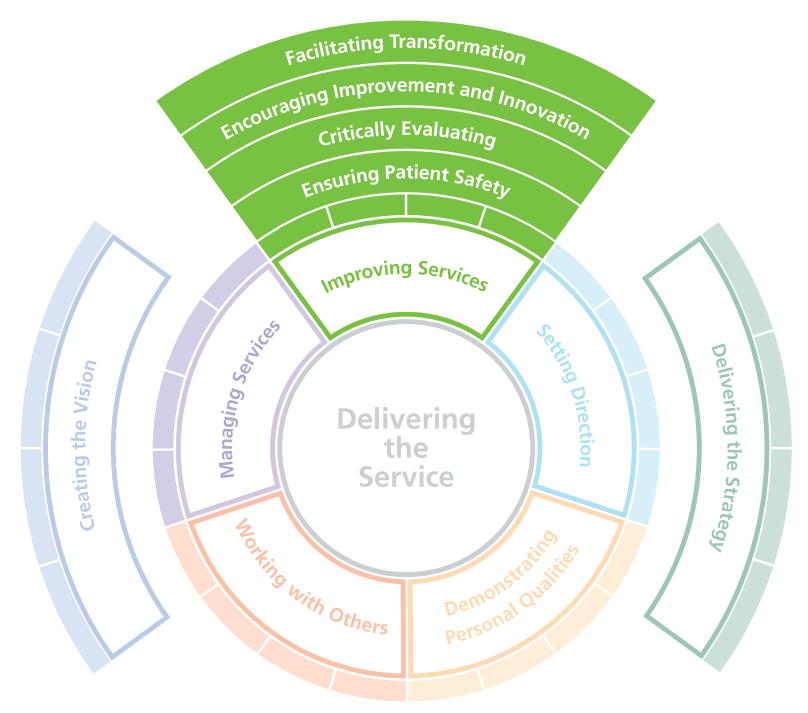

Effective leadership requires individuals to make a real difference to people's health by delivering high quality services and by developing improvements to services. To do so, they must demonstrate effective in:

- Ensuring patient safety by assessing and managing risk to patients associated with service developments, balancing economic consideration with the need for patient safety

- Critically evaluating by being able to think analytically, conceptually and to identify where services can be improved, working individually or as part of a team

- Encouraging improvement and innovation by creating a climate of continuous service improvement

- Facilitating transformation by actively contributing to change processes that lead to improving healthcare.

\subsection{Ensuring patient safety}

1. Identify and quantify the risk to patients using information from a range of sources

2. Use evidence, both positive and negative, to identify options

3. Use systematic ways of assessing and minimising risk

4. Monitor the effects and outcomes of change

4.2 Critically evaluating

1. Obtain and act on patient, carer and user feedback and experiences

2. Assess and analyse processes using up-to-date improvement methodologies

3. Identify healthcare improvements and create solutions through collaborative working

4. Appraise options, and plan and take action to implement and evaluate improvements 4.3 Encouraging improvement and innovation

1. Question the status quo

2. Act as a positive role model for innovation

3. Encourage dialogue and debate with a wide range of people

4. Develop creative solutions to transform services and care

4.4 Facilitating transformation

1. Model the change expected

2. Articulate the need for change and its impact on people and services

3. Promote changes leading to systems redesign

6. Motivate and focus a group to accomplish change 


\section{Setting Direction}

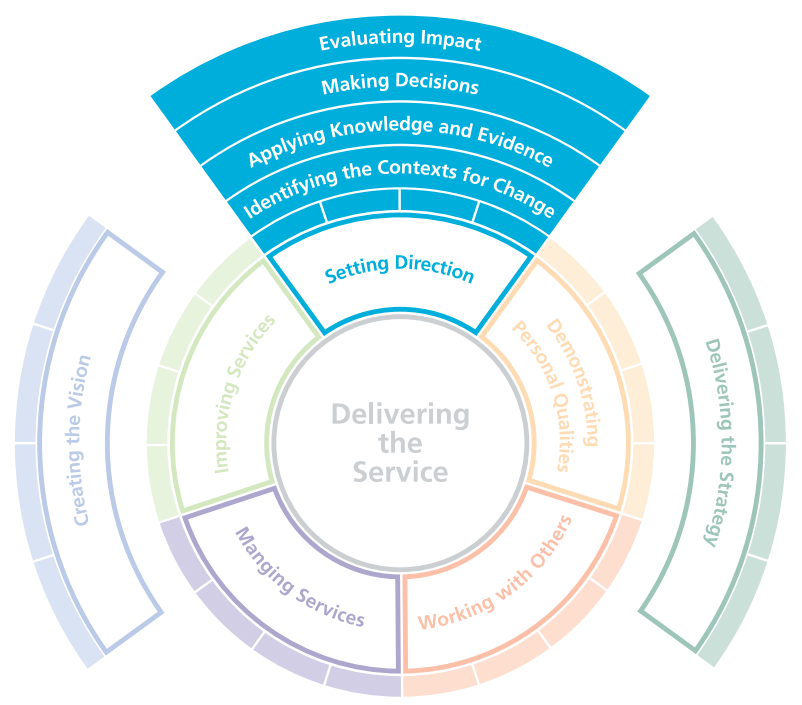

Effective leadership requires individuals to contribute to the strategy and aspirations of the organisation and act in a manner consistent with its values. To do so, they must demonstrate effective in:

- Identifying the contexts for change by being aware of the range of factors to be taken into account

- Applying knowledge and evidence by gathering information to produce an evidence-based challenge to systems and processes in order to identify opportunities for service improvements

- Making decisions using their values, and the evidence, to make good decisions

- Evaluating impact by measuring and evaluating outcomes, taking corrective action where necessary and by being held to account for their decisions.

\section{1 ldentifying the contexts for change}

1. Demonstrate awareness of the political, social, technical, economic, organisational and professional environment

2. Understand and interpret relevant legislation and accountability frameworks

3. Anticipate and prepare for the future by scanning for ideas, best practice and emerging trends that will have an impact on health outcomes

4. Develop and communicate aspirations

\subsection{Applying knowledge and evidence}

1. Use appropriate methods to gather data and information

2. Carry out analysis against an evidence-based criteria set

3. Use information to challenge existing practices and processes

4. Influence others to use knowledge and evidence to achieve best practice

\subsection{Making decisions}

1. Participate in and contribute to organisational decision-making processes

2. Act in a manner consistent with the values and priorities of their organisation and profession

3. Educate and inform key people who influence and make decisions

4. Contribute a clinical perspective to team, department, system and organisational decisions

5.4 Evaluating impact

1. Test and evaluate new service options

2. Standardise and promote new approaches

3. Overcome barriers to implementation

4. Formally and informally disseminate good practice 


\section{Creating the Vision}

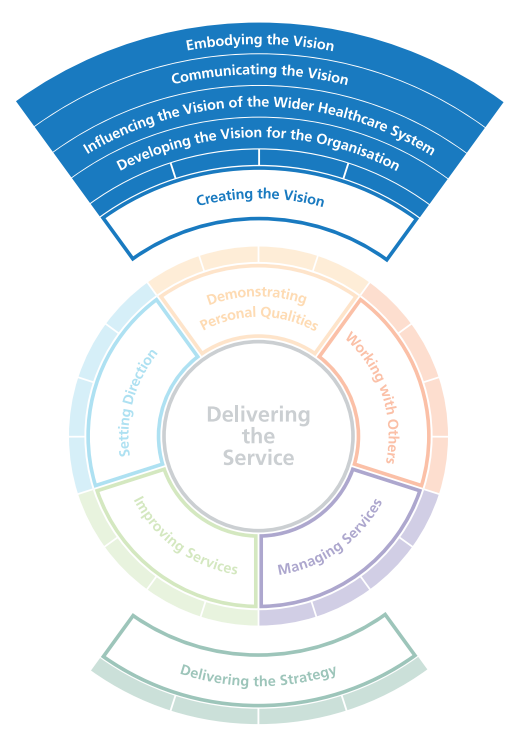

Effective leadership involves creating a compelling vision for the future, and communicating this within and across organisations. This requires individuals to demonstrate effectiveness in:

- Developing the vision of the organisation, looking to the future to determine the direction for the organisation

- Influencing the vision of the wider healthcare system by working with partners across organisations

- Communicating the vision and motivating others to work towards achieving it

- Embodying the vision by behaving in ways which are consistent with the vision and values of the organisation.

\subsection{Developing the vision for the organisation}

1. Actively engage with colleagues and key influencers, including patients and public, about the future of the organisation

2. Broadly scan and analyse the full range of factors that will impact upon the organisation, to create likely scenarios for its future

3. Create a vision which is bold, innovative and reflects the core values of the NHS

4. Continuously ensures that the organisation's vision is compatible with future developments within the wider healthcare system.

\subsection{Influencing the vision of the wider healthcare system}

1. Seek opportunities to engage in debate about the future of health and care related services

2. Work in partnership with others in the healthcare system to develop a shared vision

3. Negotiate compromises in the interests of better patient services

4. Influence key decision-makers who determine future government policy that impacts on the NHS and its services.

\subsection{Communicating the vision}

1. Communicate their ideas and enthusiasm about the future of the organisation and its services confidently and in a way which engages and inspires others

2. Express the vision clearly, unambiguously and vigorously

3. Ensure that stakeholders within and beyond the immediate organisation are aware of the vision and any likely impact it may have on them

4. Take time to build critical support for the vision and ensure it is shared and owned by those who will be communicating it.

\subsection{Embodying the vision}

1. Act as a role model, behaving in a manner which reflects the values and principles inherent in the vision

2. Demonstrate confidence, self belief, tenacity and integrity in pursuing the vision

3. Challenge behaviours which are not consistent with the vision

4. Identify symbols, rituals and routines within the organisation which are not consistent with the vision, and replace them with ones that are. 


\section{Delivering the Strategy}



Effective leadership involves delivering the strategy by developing and agreeing strategic plans that place patient care at the heart of the service, and ensuring that these are translated into achievable operational plans. This requires individuals to demonstrate effectiveness in:

- Framing the strategy by identifying strategic options for the organisation and drawing upon a wide range of information, knowledge and experience

- Developing the strategy by engaging with colleagues and key stakeholders

- Implementing the strategy by organising, managing and assuming the risks of the organisation

- Embedding the strategy by ensuring that strategic plans are achieved and sustained.

\subsection{Framing the strategy}

1. Take account of the culture, history and long term underlying issues for the organisation

2. Use sound organisational theory to inform the development of strategy

3. Identify best practice which can be applied to the organisation

4. Identify strategic options which will deliver the organisation's vision

\subsection{Developing the strategy}

1. Engage with key individuals and groups to formulate strategic plans to meet the vision

2. Strive to understand others' agendas, motivations and drivers in order to develop strategy which is sustainable

3. Create strategic plans which are challenging yet realistic and achievable

4. Identify and mitigate uncertainties and risks associated with strategic choices

\subsection{Implementing the strategy}

1. Ensure that strategic plans are translated into workable operational plans, identifying risks, critical success factors and evaluation measures

2. Identify and strengthen organisational capabilities required to deliver the strategy

3. Establish clear accountability for the delivery of all elements of the strategy, hold people to account and expect to be held to account themselves

4. Respond quickly and decisively to developments which require a change in strategy

\subsection{Embedding the strategy}

1. Support and inspire others responsible for delivering strategic and operational plans, helping them to overcome obstacles and challenges, and to remain focused

2. Create a consultative organisational culture to support delivery of the strategy and to drive strategic change within the wider healthcare system

3. Establish a climate of transparency and trust where results are discussed openly

4. Monitor and evaluate strategic outcomes, making adjustments to ensure sustainability of the strategy 







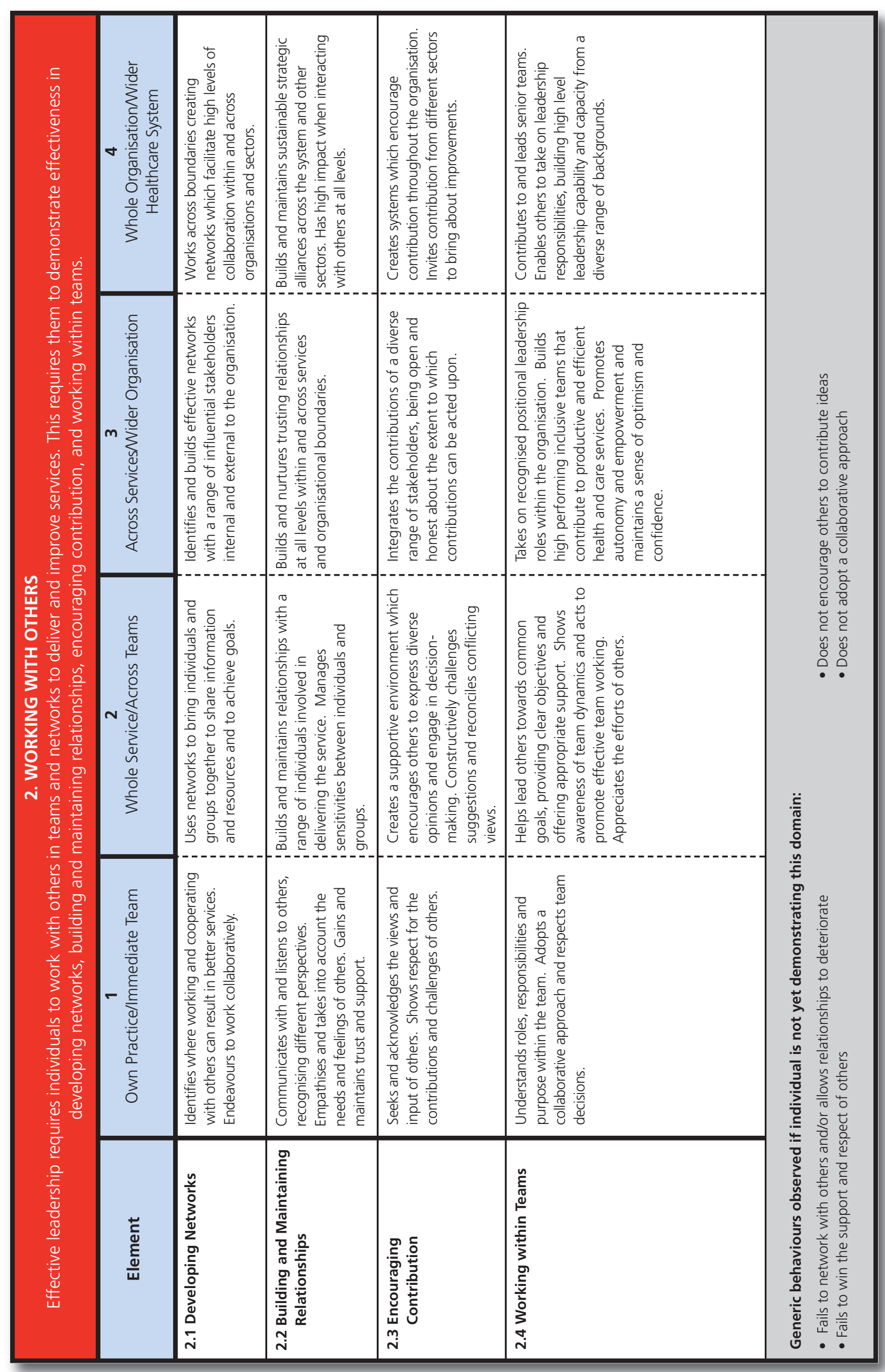














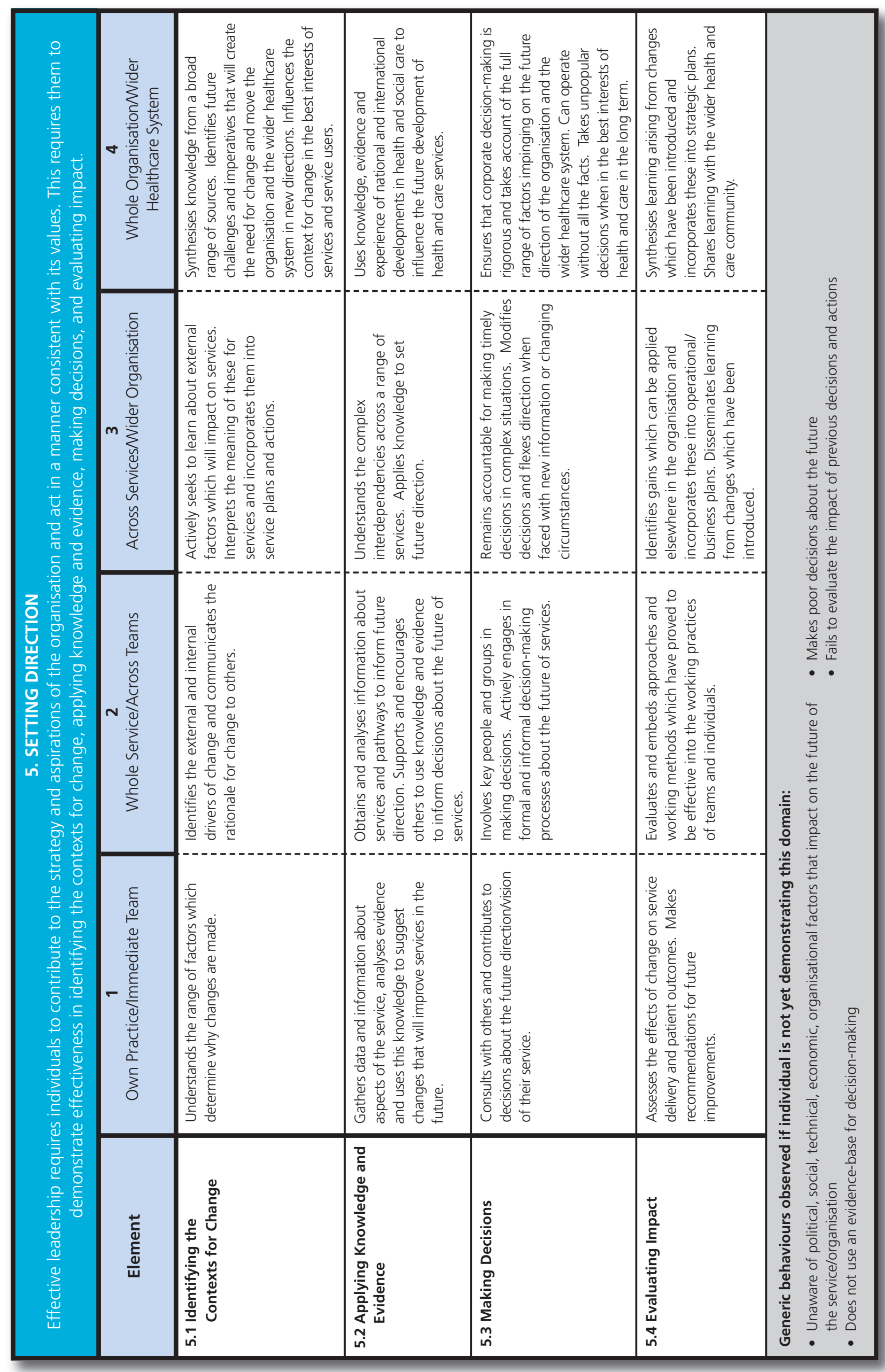




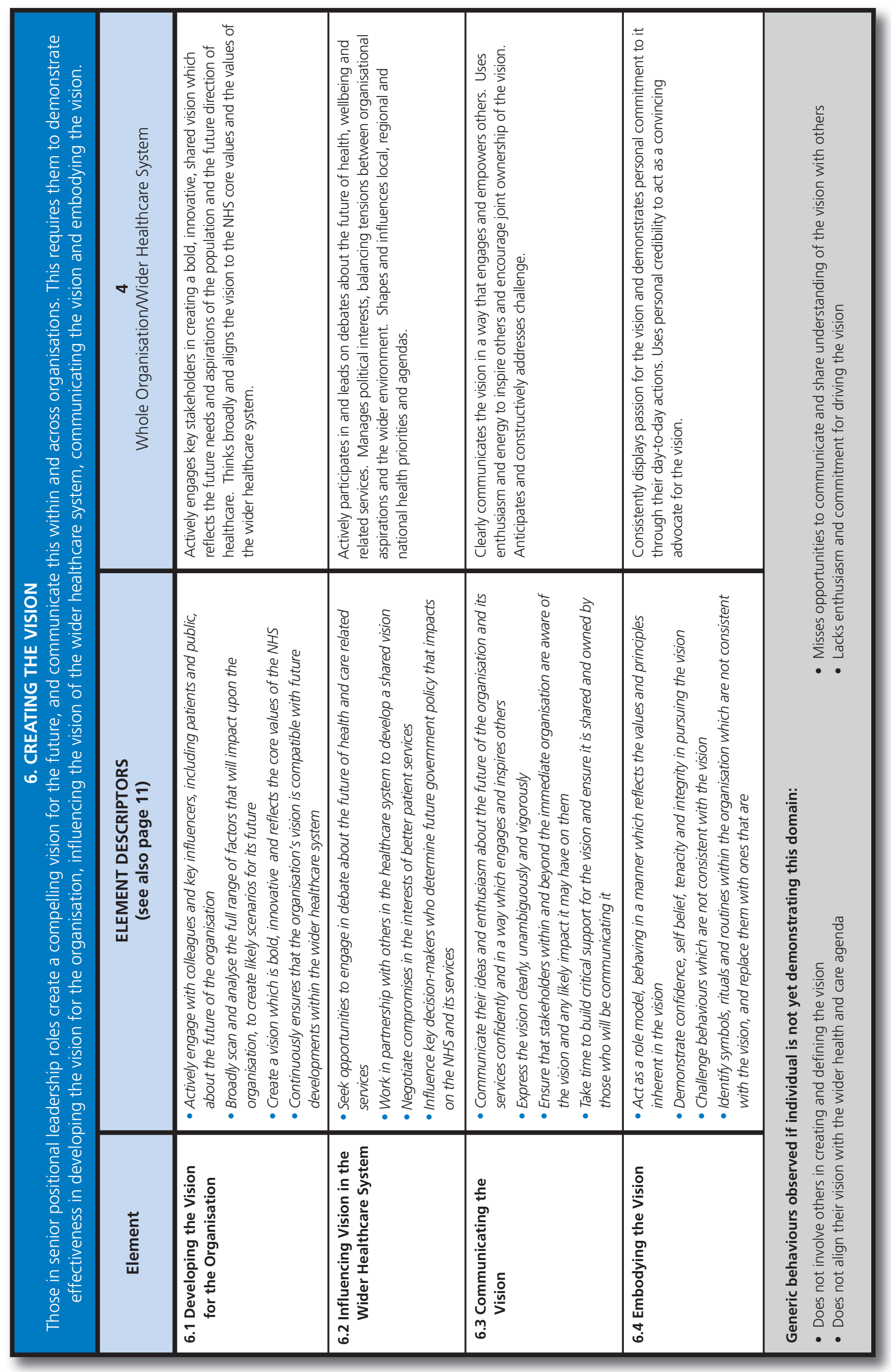




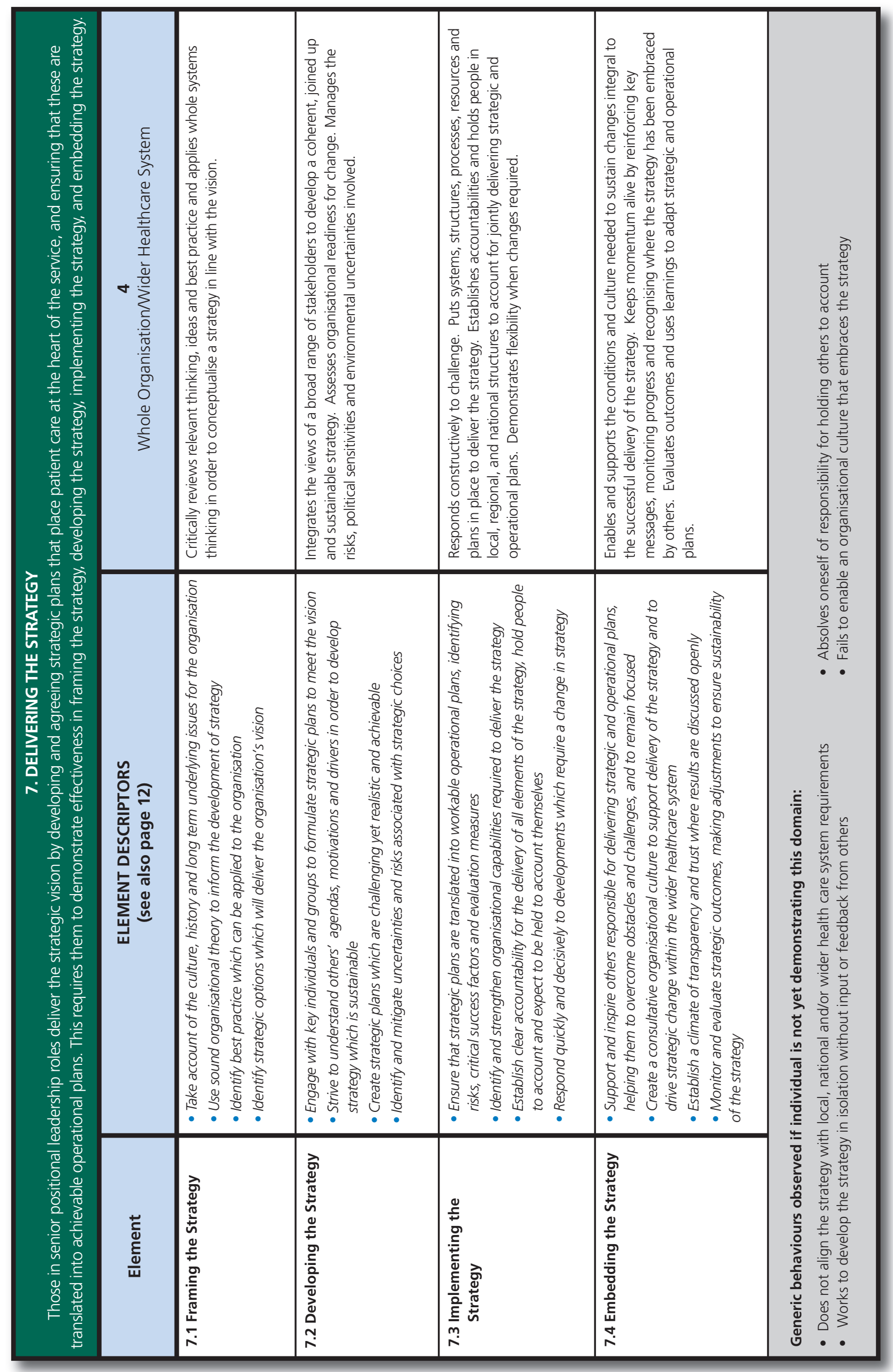


Leadership Academy 